\title{
Discovery of magnetic fields in hot subdwarfs ${ }^{\star}$
}

\author{
S. J. O’'Toole ${ }^{1}$, S. Jordan ${ }^{2}$, S. Friedrich ${ }^{3}$, and U. Heber ${ }^{1}$ \\ ${ }^{1}$ Dr. Remeis-Sternwarte, Astronomisches Institut der Universität Erlangen-Nürnberg, Sternwartstr. 7, Bamberg 96049, \\ Germany \\ e-mail: otoole@sternwarte.uni-erlangen.de \\ 2 Astronomisches Rechen-Institut, Mönchhofstr. 12-14, 69221 Heidelberg, Germany \\ ${ }^{3}$ Max-Planck-Institut für extraterrestrische Physik, Giessenbachstr., 85748 Garching, Germany
}

Received 16 December 2004 / Accepted 4 March 2005

\begin{abstract}
We present polarisation measurements of sdB and sdO stars using FORS1 on the VLT. The observations were made as part of a project to determine whether magnetic fields in two super-metal-rich stars can explain their extreme abundance peculiarities. Field strengths of up to $\sim 1.5 \mathrm{kG}$ range have been measured at varying levels of significance in each of our six targets, however no clear evidence was found between apparently normal subdwarfs and the metal-rich objects. The origin of the magnetic fields is unknown. We also discuss the implications of our measurements for magnetic flux conservation in late stages of stellar evolution.
\end{abstract}

Key words. stars: subdwarfs - stars: magnetic fields

\section{Introduction}

The measurement of magnetic fields in evolved stars at the kilogauss level has become possible only recently, when spectropolarimeters attached to telescopes with diameters larger than $8 \mathrm{~m}$ have become available. The implementation of circularlypolarised spectropolarimetry with FORS1 spectrograph of the Very Large Telescope (VLT) has opened the door to study magnetic fields in a large range of stars. The technique was first described by Bagnulo et al. (2002), who used the hydrogen Balmer lines to measure a mean longitudinal field of $\sim 2 \mathrm{kG}$ in a chemically peculiar A star. Such low magnetic fields have now been detected in white dwarfs and central stars of planetary nebulae (Aznar Cuadrado et al. 2004; Jordan et al. 2005).

Hot subdwarfs are subluminous objects that dominate the population of faint blue stars in our own galaxy. The subdwarf B stars (sdBs) have hydrogen-rich atmospheres with effective temperatures below about $40000 \mathrm{~K}$ (e.g. Heber 1986). They are also typically helium-poor. Subdwarf O (sdO) stars on the other hand cover a much larger range of atmospheric compositions with a large spread of hydrogen and helium abundances. Their effective temperatures are in the range between 40000 and $90000 \mathrm{~K}$. In the $\log g-T_{\text {eff }}$ diagram they are found close to ("born-again") post-AGB or post-extreme horizontal branch tracks (e.g. Husfeld et al. 1989; Ströer et al. 2005). Both groups are believed to be the progeny of $1-2 \%$ of the white dwarfs. Recently, two subdwarf B stars (UVO 0512-08 and PG 0909+276) were discovered to have unusually high

^ Based on observations collected at the European Southern Observatory, Paranal, Chile, under programme ID 072.D-0290(A). abundances of iron-group elements, e.g. these stars have up to $10^{5}$ times more vanadium and nickel than the Sun (Edelmann et al. 2001). While no direct evidence was found in their spectra, one obvious possibility is that these anomalously high abundances are somehow related to large magnetic fields, as seen in the chemically peculiar A stars. Inspired by this discovery, we started a project to measure magnetic fields in hot subdwarfs.

There is only one published study of a search for magnetic fields in sdB and sdO stars, which was carried out using the Special Astronomical Observatory $6 \mathrm{~m}$ telescope (Elkin 1996). The author inferred a longitudinal magnetic field of $-1690 \pm 60 \mathrm{G}$ (based on three measurements of the polarisation in the He I $5875 \AA$ line of the $\mathrm{sdO} \mathrm{BD}+75^{\circ} 325(V=9.21)$ and a variable field in the sdOB Feige $66(V=10.64)$ with measurements of $-1300 \pm 320 \mathrm{G}$ (He I 5875), $-1300 \pm 580$ (He I 5875), and $+1750 \pm 230 \mathrm{G}(\mathrm{H} \alpha$, He I 5875), and $+460 \mathrm{G}(\mathrm{H} \alpha)$, each value based on one or two measurements. The uncertainties were (probably) calculated from the deviations of successive observations without taking into account individual errors in measurements or sytematic errors.

In this paper we present spectropolarimetric observations of sdBs and sdOs based on polarisation measurements using the Balmer lines and He I and II lines.

\section{Observations and reduction}

Circular polarisation spectra of six hot subdwarfs were obtained using the VLT/UT1 (Antu) with the FORS1 spectrograph on February 23, 2004 (UT) in visitor mode. FORS1 is a multi-mode focal reducer imager and grism spectrograph 
Table 1. List of targets observed. Super-metal-rich objects are marked with an asterisk.

\begin{tabular}{|c|c|c|c|c|c|c|c|c|c|c|c|}
\hline Target & $\begin{array}{c}\alpha \\
(\mathrm{J} 2000)\end{array}$ & $\begin{array}{c}\delta \\
(\mathrm{J} 2000)\end{array}$ & $\begin{array}{c}V \\
(\mathrm{mag})\end{array}$ & $\begin{array}{c}\text { UT } \\
\text { start }\end{array}$ & $\begin{array}{l}t_{\text {exp }} \\
(\mathrm{s})\end{array}$ & $\begin{array}{c}n \\
(\mathrm{~K})\end{array}$ & $T_{\text {eff }}$ & $\log g$ & $\log (\mathrm{He} / \mathrm{H})$ & sp. type & Ref. \\
\hline UVO 0512-08* & 051444.0 & -084804 & 11.3 & $00: 10: 56$ & 440 & 3 & 38800 & 5.5 & -0.8 & $\mathrm{sdB}$ & 1 \\
\hline $\mathrm{CPD}-64^{\circ} 481$ & 054759.3 & -642303 & 11.3 & 01:14:00 & 440 & 3 & 27500 & 5.6 & -2.9 & $\mathrm{sdB}$ & 2 \\
\hline PG $0909+276^{*}$ & 091251.7 & +272031 & 12.1 & $02: 16: 27$ & 1200 & 3 & 36900 & 5.9 & -0.8 & $\mathrm{sdB}$ & 1 \\
\hline$C D-31^{\circ} 4800$ & 073630.2 & -321244 & 10.6 & $04: 54: 30$ & 240 & 3 & 44000 & 5.9 & $\sim 1.5$ & $\mathrm{sdO}$ & 4 \\
\hline HD 76431 & 085611.2 & +014038 & 9.2 & $05: 39: 56$ & 110 & 9 & 31000 & 4.5 & -1.5 & $\operatorname{sdB}$ & 3 \\
\hline LSE 153 & 135308.2 & -464342 & 11.4 & $06: 44: 52$ & 550 & 9 & 70000 & 4.75 & $\sim 2$ & $\mathrm{sdO}$ & 5 \\
\hline
\end{tabular}

References: (1) Edelmann et al. (2001); (2) this work; (3) Ramspeck et al. (2001); (4) Bauer \& Husfeld (1995); (5) Husfeld et al. (1989).

equipped with a Wollaston prism and rotatable retarder plate mosaics in the parallel beam allowing linear and circular polarimetry and spectropolarimetry. We used the 600B grism, covering the spectral range $3400-5900 \AA$, with a $0.5^{\prime \prime}$ slit, leading to a spectral resolution of $2.8 \AA$. Details about our targets can be found in Table 1. Two out of six of them are the supermetal-rich sdB stars found by Edelmann et al. (2001), one is an apparently normal $\mathrm{sdB}$, one is a post-EHB sdB, while the last two stars are sdOs. The two metal-rich objects were observed in the first half of the night, and subsequent bad weather meant that more "normal" abundance targets could not be observed.

Bias frames, flat-fields and $\mathrm{He}+\mathrm{HgCd}$ arc spectra were taken at the end of the night. The data were reduced using standard IRAF routines for bias subtraction, cosmic ray removal and flat fielding. After the flat-field correction, the stellar spectra were extracted from each frame by summing up all CCD rows for the $e$ and $o$ beams. Background sky light was averaged over 10 rows on either side of the object spectrum (giving a total of 20 rows) and subtracted.

As noted by Jordan et al. (2005), it is very important to make sure that the spectra are correctly wavelength calibrated; an incorrect calibration might lead to a spurious polarisation signal. Calibration was done independently for the spectra of each beam ( $e$ and $o$ ) and each position of the retarder plate $\left( \pm 45^{\circ}\right)$. The wavelength accuracy of each spectrum is about $3 \mathrm{~km} \mathrm{~s}^{-1}$ or $0.05 \AA$ at $\mathrm{H} \beta$, which is much lower than the spectral resolution. Stokes $I$ spectra (i.e. unpolarised, the sum of the $e$ and $o$ beams) are shown in Fig. 1 with the continuum normalised to unity and each spectrum offset by 0.5 (not scaled) for clarity. Note that the top two spectra are the helium-rich sdO stars, the two middle spectra are the super-metal-rich sdBs and those at the bottom are the "normal" abundance sdBs.

To derive the level of circular polarisation from such a sequence, we added the exposures taken with the same quarterwave plate angle. Stokes $V / I$ can then be found using

$\frac{V}{I}=\frac{R-1}{R+1}$, with $R^{2}=\left(\frac{f_{\mathrm{o}}}{f_{\mathrm{e}}}\right)_{\alpha=+45} \times\left(\frac{f_{\mathrm{e}}}{f_{\mathrm{o}}}\right)_{\alpha=-45}$

where $V$ is the Stokes parameter describing the net circular polarisation, $I$ is the unpolarised intensity, $\alpha$ indicates the nominal value of the position angle of the retarder-wave plate, and $f_{\mathrm{o}}$ and $f_{\mathrm{e}}$ are the fluxes on the detector from the ordinary and extraordinary beams of the Wollaston prism, respectively.

The mean longitudinal field can be derived using the weak field approximation, which is valid in the presence of instrumental broadening, but not in the case of rotational broadening.

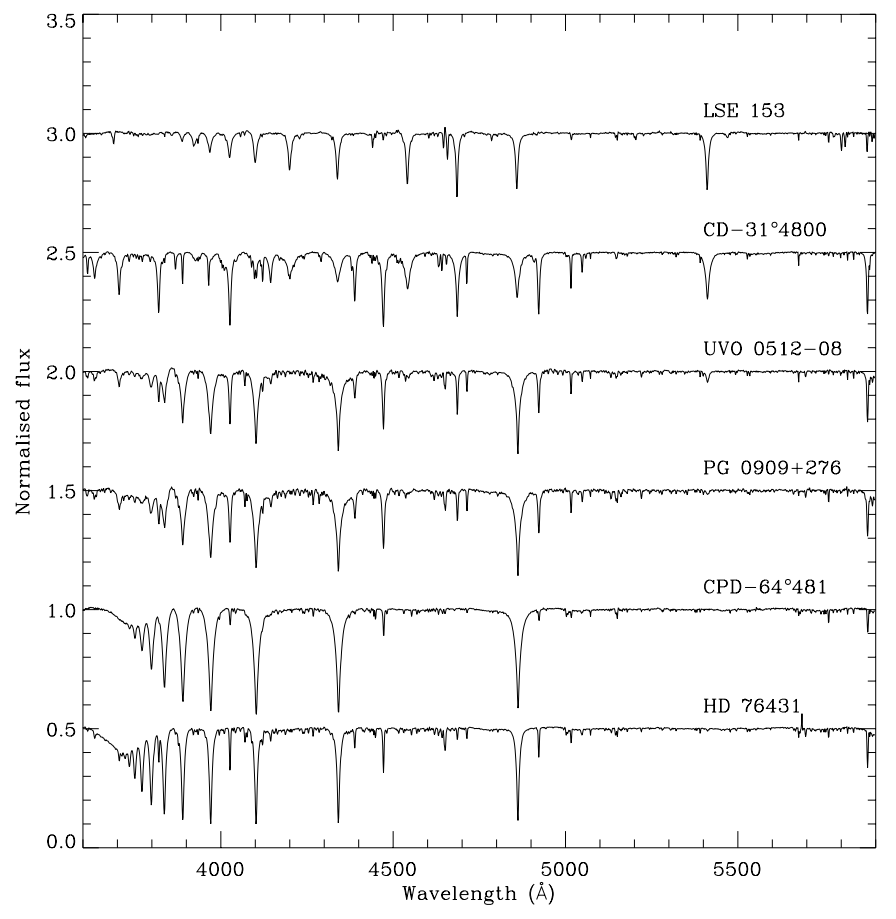

Fig. 1. Normalised spectra of our six targets. The spectra are offset by 0.5 for clarity.

The limiting value of $v \sin i$ is dependent on the intrinsic line width; the Balmer lines are not expected to be affected because Stark broadening is dominant. For example, if the line width is $\sim 15 \AA$ (typical for the Balmer lines in hot subdwarfs), $v \sin i$ would have to be greater than $1000 \mathrm{~km} \mathrm{~s}^{-1}$, while a narrow line with width $3 \AA$ would be useless if $v \sin i>200 \mathrm{~km} \mathrm{~s}^{-1}$. None of our targets are known to be rapid rotators in any case. The field strength is compared with the Stokes $V / I$ spectrum using the following equation (Landstreet 1982):

$\frac{V}{I}=-g_{\mathrm{eff}} \frac{e}{4 \pi m_{\mathrm{e}} c^{2}} \lambda^{2} \frac{1}{I_{\lambda}} \frac{\mathrm{d} I}{\mathrm{~d} \lambda}\left\langle B_{z}\right\rangle$

where $g_{\text {eff }}$ is the effective Landé factor, $\lambda$ is wavelength in $\AA$, $\left\langle B_{z}\right\rangle$ is the mean longitudinal field in Gauss, and $e /\left(4 \pi m_{\mathrm{e}} c^{2}\right) \simeq$ $4.67 \times 10^{-13} \mathrm{G}^{-1} \AA^{-1}$. Note that we are only measuring the longitudinal field average over the stellar surface; the maximum field strength may be larger.

The longitudinal component of the magnetic field for each measurement was determined by comparing the observed circular polarisation for an interval of $\pm 20 \AA$ around the strongest 
Table 2. Effective Landé factors for the observed He I and He II lines, along with the next three strongest lines that may be observed with a different FORS1 grism.

\begin{tabular}{|c|c|c|c|c|c|c|c|c|c|}
\hline \multicolumn{10}{|c|}{$\mathrm{HeI}$} \\
\hline \multirow[t]{2}{*}{ Line } & \multirow[t]{2}{*}{$g_{\text {eff }}$} & \multicolumn{4}{|c|}{ Lower level } & \multicolumn{4}{|c|}{ Upper level } \\
\hline & & $J$ & $L$ & $S$ & $g$ & $J$ & $L$ & $S$ & $g$ \\
\hline $4026.29 \AA$ & 1.17 & 2 & 1 & 1 & $3 / 2$ & 3 & 2 & 1 & $4 / 3$ \\
\hline $4471.60 \AA$ & 1.17 & 2 & 1 & 1 & $3 / 2$ & 3 & 2 & 1 & $4 / 3$ \\
\hline $4713.28 \AA$ & 1.25 & 2 & 1 & 1 & $3 / 2$ & 1 & 0 & 1 & 2 \\
\hline $4921.94 \AA$ & 1.00 & 1 & 1 & 0 & 1 & 2 & 2 & 0 & 1 \\
\hline $5015.69 \AA$ & 1.00 & 0 & 0 & 0 & - & 1 & 1 & 0 & 1 \\
\hline $5047.74 \AA$ & 1.00 & 1 & 1 & 0 & 1 & 0 & 0 & 0 & - \\
\hline $5875.83 \AA$ & 1.17 & 2 & 1 & 1 & $3 / 2$ & 3 & 2 & 1 & $4 / 3$ \\
\hline $6678.16 \AA$ & 1.00 & 1 & 1 & 0 & 1 & 2 & 2 & 0 & 1 \\
\hline $7065.53 \AA$ & 1.25 & 2 & 1 & 1 & $3 / 2$ & 1 & 0 & 1 & 2 \\
\hline $7281.37 \AA$ & 1.00 & 1 & 1 & 0 & 1 & 0 & 0 & 0 & - \\
\hline \multicolumn{10}{|c|}{ He II } \\
\hline \multirow[t]{2}{*}{ Line } & \multirow{2}{*}{$g_{\text {eff }}$} & \multicolumn{4}{|c|}{ Lower level } & \multicolumn{4}{|c|}{ Upper level } \\
\hline & & $\bar{J}$ & $L$ & $S$ & $g$ & $\bar{J}$ & $L$ & $S$ & $g$ \\
\hline $4100.05 \AA$ & 1.06 & 3.5 & 3 & 0.5 & 1.14 & 4.5 & 4 & 0.5 & 1.1 \\
\hline $4199.84 \AA$ & 1.06 & 3.5 & 3 & 0.5 & 1.14 & 4.5 & 4 & 0.5 & 1.1 \\
\hline $4338.68 \AA$ & 1.06 & 3.5 & 3 & 0.5 & 1.14 & 4.5 & 4 & 0.5 & 1.1 \\
\hline $4541.59 \AA$ & 1.06 & 3.5 & 3 & 0.5 & 1.14 & 4.5 & 4 & 0.5 & 1.1 \\
\hline $4685.90 \AA$ & 1.07 & 2.5 & 2 & 0.5 & 1.2 & 3.5 & 3 & 0.5 & 1.14 \\
\hline $4859.32 \AA$ & 1.06 & 3.5 & 3 & 0.5 & 1.14 & 4.5 & 4 & 0.5 & 1.1 \\
\hline $5411.51 \AA$ & 1.06 & 3.5 & 3 & 0.5 & 1.14 & 4.5 & 4 & 0.5 & 1.1 \\
\hline $6560.09 \AA$ & 1.06 & 3.5 & 3 & 0.5 & 1.14 & 4.5 & 4 & 0.5 & 1.1 \\
\hline
\end{tabular}

absorption lines with the prediction of Eq. (2). As in Aznar Cuadrado et al. (2004) and Jordan et al. (2005), we determined $\left\langle B_{z}\right\rangle$ using a $\chi^{2}$-minimisation procedure. Unlike the case of central stars of planetary nebulae, systematic errors due to the blending of Balmer lines and the Pickering series He II lines should be small. This is because in the stars where the hydrogen lines are strong, the helium lines are not strong, and vice versa. In the case of strong Balmer lines, some of the helium lines are very weak. The strongest are He I 5875, He I 4471, He I 4921 and He II 4686. We used the criterion that the line must be stronger than $10 \%$ below the continuum to be measurable. Because of this, only in the two He-rich sdO stars could other helium lines be used. Other systematic errors besides the blending effect are difficult to estimate in strength, but we believe that they are lower than $200 \mathrm{G}$.

\section{Landé factors}

Previous spectropolarimetric studies using Balmer or He II lines have not needed to consider the effect of the Landé $g$-factor on the magnetic field strength. This is because for these lines $g_{\text {eff }}$ is approximately unity. This is not the case, however, for many of the He I lines that we can measure in our subdwarfs. We have determined the effective Landé factors with the classical formula (e.g. Leone et al. 2000).

$$
\begin{aligned}
g_{\mathrm{eff}}= & 0.5\left(g_{\text {low }}+g_{\text {up }}\right)+0.25\left(g_{\text {low }}-g_{\text {up }}\right) \\
& \times\left(J_{\text {low }}\left(J_{\text {low }}+1\right)-J_{\text {up }}\left(J_{\text {up }}+1\right)\right)
\end{aligned}
$$

where $g_{\text {low }}$ and $g_{\text {up }}$ are the Landé factors of the lower and upper energy level. As far as the line is an electric dipole line this formula holds independently of the coupling scheme $(\mathrm{L}-\mathrm{S}, \mathrm{j}-\mathrm{j}$ or intermediate coupling) for the atomic levels involved. For calculating $g_{\text {low }}$ and $g_{\text {up }}$ we used the approximation valid for L-S coupling:

$g=1+\frac{J(J+1)-L(L+1)+S(S+1)}{2 J(J+1)}$

where $J, L$, and $S$ are the total, the orbital, and the spin angular momentum quantum numbers of the respective energy levels. For hydrogen Balmer lines $g_{\text {eff }}=1$ was used, although we are aware that it is slightly different from unity. However, the deviation from unity is less than $7 \%$ for all lines of the Balmer series (Casini \& Landi degl'Innocenti 1994) which is negligible for our purposes. For the same reason, we set $g_{\mathrm{eff}}=1$ for the He II lines. The Landé factors of all helium lines observed are shown in Table 2, along with three strong lines that may be observable using a redder FORS1 grism. Note that all He I transitions that have $S=0$ have $g_{\text {eff }}=1$, while $S=1$ transitions have $g_{\text {eff }}>1$.

\section{Results}

The results of our analysis are shown in Table 3 with results for each line measured, along with the weighted average field strength. The values shown here must be intrinsic to the star (and not e.g. interstellar or instrumental), since no polarisation effects are visible in the continuum. The line errors are simply a product of the $\chi^{2}$ test (see Aznar Cuadrado et al. 2004; and Jordan et al. 2005, for details), while the error in the average field strength was calculated using $\sigma=\sum 1 / \sigma_{i}^{-1 / 2}$, where the 
Table 3. Mean longitudinal field strengths (in Gauss) measured from all lines that satisfied our $10 \%$ below the continuum criterion. No entry means the line is either not present, or does not satisfy the criterion.

\begin{tabular}{ccccccc}
\hline \hline Line & UVO 0512-08 & ${\text { CPD }-64^{\circ} 481}^{2}$ & PG 0909+276 & HD 76431 & CD -31 4800 & LSE 153 \\
& $B_{z}(\mathrm{G})$ & $B_{z}(\mathrm{G})$ & $B_{z}(\mathrm{G})$ & $B_{z}(\mathrm{G})$ & $B_{z}(\mathrm{G})$ & $B_{z}(\mathrm{G})$ \\
\hline H $\beta$ & $-826 \pm 338$ & $-1208 \pm 266$ & $-2596 \pm 475$ & $-1405 \pm 175$ & - & - \\
H $\gamma$ & $-2024 \pm 603$ & $201 \pm 406$ & $-1095 \pm 550$ & $-1021 \pm 178$ & - & - \\
He I 4471 & $-1915 \pm 533$ & - & $-1323 \pm 480$ & $-1293 \pm 293$ & $-1356 \pm 324$ & - \\
He I 4921 & $-718 \pm 415$ & - & $-478 \pm 851$ & $-1083 \pm 435$ & $-1665 \pm 454$ & - \\
He I 5015 & - & - & - & - & $-481 \pm 472$ & - \\
He I 5875 & $-1397 \pm 379$ & $-718 \pm 745$ & $-552 \pm 535$ & $-650 \pm 170$ & $-545 \pm 357$ & - \\
He I 4713 & - & - & - & - & $-1562 \pm 468$ & - \\
He II 4686 & $-1473 \pm 362$ & - & $-750 \pm 926$ & - & $-753 \pm 418$ & $-1329 \pm 432$ \\
He II 5412 & - & - & - & - & $125 \pm 641$ & $732 \pm 466$ \\
He II 4859 & - & - & - & - & $-1016 \pm 941$ & $-1678 \pm 418$ \\
He II 4541 & - & - & - & - & $-2692 \pm 951$ & $-2047 \pm 472$ \\
He II 4339 & - & - & - & - & $-1617 \pm 1640$ & $-1013 \pm 904$ \\
He II 4200 & - & - & $-1448 \pm 222$ & $-1096 \pm 91$ & $-1050 \pm 161$ & $-1128 \pm 212$ \\
\hline Average & $-1306 \pm 161$ & $-885 \pm 207$ & -1448 &
\end{tabular}

Table 4. For $B_{z}=0,600$, and $1200 \mathrm{G}$ we produced 1000 simulated polarisation spectra with the same noise level as the programme stars and used the same spectral lines as in the fits of Table 3. We list the weighted means and standard deviations (from all spectral lines) of the fits at each input $B_{z}$ and additionally the smallest and largest value from all fits.

\begin{tabular}{lrrrrrr}
\hline \hline $\begin{array}{l}\text { input } B_{z} \\
(\mathrm{G})\end{array}$ & $\mathrm{UVO} 0512-08$ & $\mathrm{CPD}-64^{\circ} 481$ & PG 0909+276 & HD 76431 \\
$(\mathrm{G})$ & $(\mathrm{G})$ & $\begin{array}{r}\text { CD }-31^{\circ} 4800 \\
(\mathrm{G})\end{array}$ & $\begin{array}{r}\text { LSE 153 } \\
(\mathrm{G})\end{array}$ \\
\hline 0, average & $-5 \pm 188$ & $3 \pm 300$ & $18 \pm 266$ & $4 \pm 128$ & $0 \pm 163$ & $-1 \pm 239$ \\
0, smallest & -513 & -862 & -792 & -446 & -554 & -685 \\
0, largest & 646 & +831 & +1035 & +393 & +540 & +857 \\
600, average & $550 \pm 184$ & $582 \pm 294$ & $555 \pm 269$ & $578 \pm 125$ & $539 \pm 170$ & $603 \pm 243$ \\
600, smallest & -62 & -387 & -249 & +233 & -699 & -250 \\
600, largest & +1108 & +1692 & +1603 & +964 & +1178 & +1346 \\
1200, average & $1106 \pm 186$ & $1170 \pm 291$ & $1119 \pm 266$ & $1131 \pm 130$ & $1090 \pm 170$ & $1194 \pm 243$ \\
1200, smallest & +490 & +164 & +185 & 614 & +507 & +489 \\
1200, largest & +1740 & +2196 & +2016 & +1505 & +1786 & 1842 \\
\hline
\end{tabular}

$\sigma_{i}$ are the individual line errors. To the untrained eye, it appears that the results for individual lines are internally inconsistent. This will be discussed below, as will the reality of our errors, and the statistical significance of field strengths we have measured.

\subsection{Statistical significance of our measurements}

As was already demonstrated by Aznar Cuadrado et al. (2004) for white dwarfs and Jordan et al. (2005) for central stars of planetary nebulae, even a small polarisation signature, slightly lower than the noise level of the spectro-polarimetric data, can be detected when several spectral lines (and multiple observations) are used. We again investigated this with simulated data of the same noise level than in the subdwarf observations.

For this purpose we produced simulated polarisation spectra for assumed magnetic fields between $0 \mathrm{G}$ and $+1200 \mathrm{G}$ and steps of $200 \mathrm{G}$. To these polarisation spectra we added Gaussian noise with the same standard deviation as in the observations of the respective subdwarfs. We assume Gaussian noise since we expect the CCDs to be linear and that photon noise is the dominating limitation. The difference between
Poisson and Gaussian noise is negligible at such high $S / N$, so we believe that our approximation is justified on theoretical grounds. We have also verified this by examining the frequency distribution of the noise. For each of the assumed magnetic fields we made 1000 simulations and calculated the magnetic fields of the fits from the same spectral lines we have used for the observations of each star. In Table 4 we list the results for $0 \mathrm{G},+600 \mathrm{G}$, and $+1200 \mathrm{G}$ and provided the mean result, the standard deviation, and the smallest and largest value. This should give us an impression of how realistic our statistical errors from the $\chi^{2}$ analyses are. In this table we present only the mean field strength derived from our simulations, not the value for individual lines. It is important to note, however, that the apparently internally inconsistent line-by-line results are also seen in our simulations. This occurs because $(\mathrm{d} I / \mathrm{d} \lambda) / I$ in Eq. (2) is nonlinear, meaning the noise deviations do not translate linearly into $V / I$ deviations. Put simply, the weighted average field strengths are more well defined.

Firstly, the mean results of the respective 1000 simulations are very close to the predescribed magnetic fields with deviations up to $100 \mathrm{G}$ for an assumed value of $1200 \mathrm{G}$. Secondly, the standard deviations are close to the $1 \sigma$ errors determined from the $\chi^{2}$ fits. 


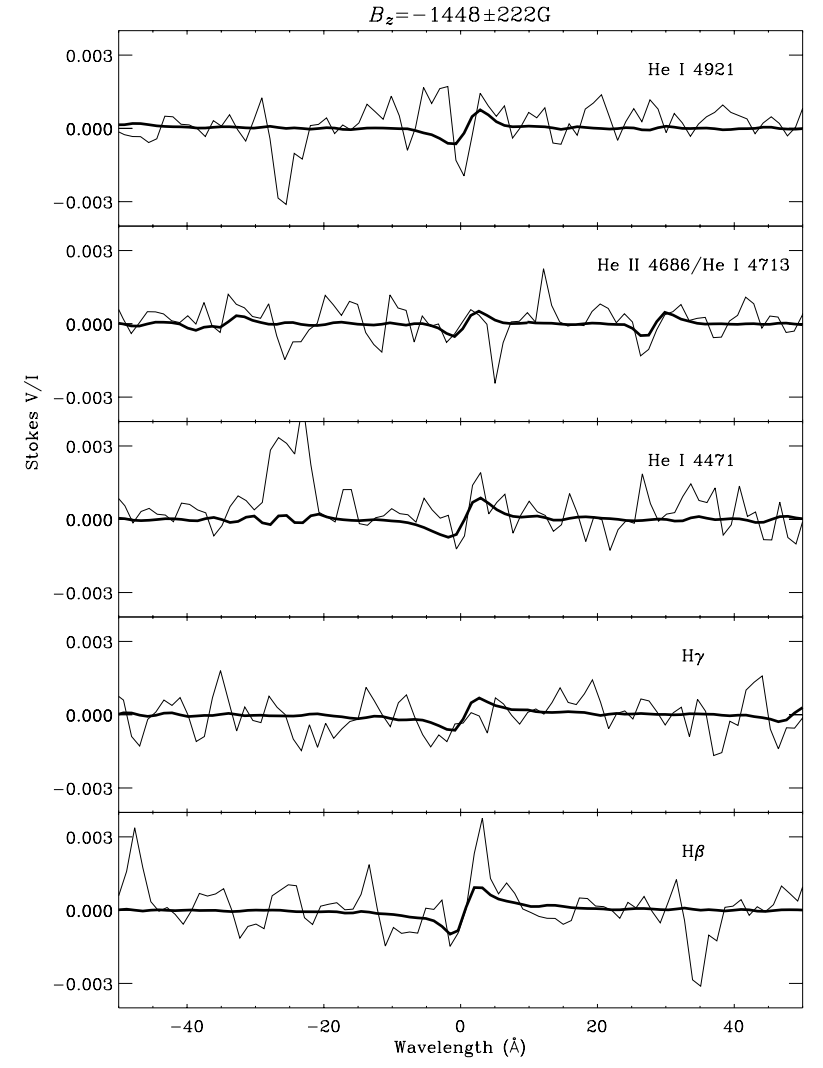

Fig. 2. Observed Stokes V/I spectra for PG 0909+276 for various hydrogen and helium lines, with value predicted by Eq. (2) overlaid (thick line). Note that the He I $4713 \AA$ line (second panel from the top) has a Landé factor $\sim 20 \%$ larger than the He II $4686 \AA$ line.

Moreover, the smallest and largest values from all 1000 fits for an assumed field of $0 \mathrm{G}$ provides a very conservative estimate of what magnetic field can be mimicked by pure noise: in all cases the fitted field strengths are larger in their absolute value than these extreme numbers; only in the case of CPD $-64^{\circ} 481$ the derived magnetic field strength of $B_{z}=$ $-885 \mathrm{G}$ comes close to the simulated extreme deviation from zero of $-862 \mathrm{G}$. Therefore, we conclude that all of our results are incompatible with $B_{z}=0$, with a somewhat smaller significance for CPD $-64^{\circ} 481$. Two of the objects (UVO 0512-08 and HD 76431) are even incompatible with $B_{z}=600 \mathrm{G}$. This indicates that our $1 \sigma$ errors are very realistic and that all stars very probably have a magnetic field. Note also that our error ranges are much smaller than for the stars studied by both Aznar Cuadrado et al. (2004) and Jordan et al. (2005), in part because we often have more spectral lines available to measure and because some of the objects are much brighter. The individual objects are discussed in more detail below, and their Stokes $V / I$ spectra are shown in Figs. 2-7.

\subsection{Super-metal-rich sdBs}

These stars are the super-metal-rich sdOB objects discovered by Edelmann et al. (2001), so we are particularly interested in their magnetic field strengths. As can be seen from Table 3, we measure $B_{z}=-1306 \pm 161 \mathrm{G}$ for UVO 0512-08 and

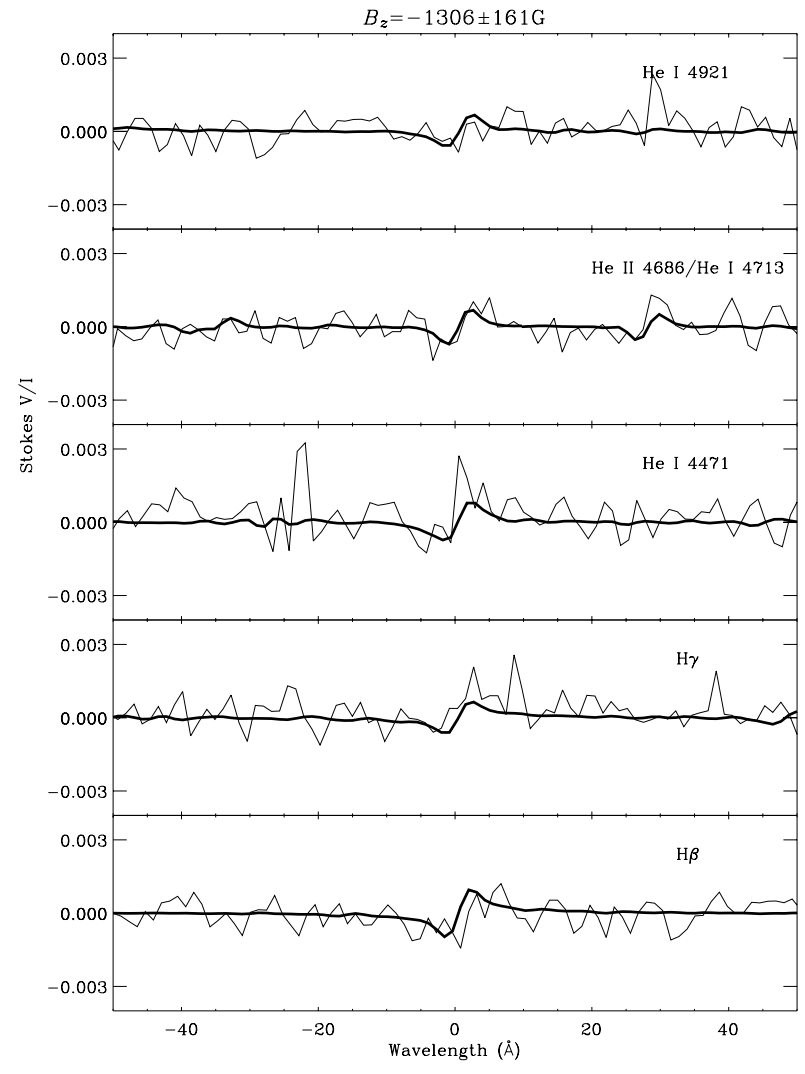

Fig. 3. Similar to Fig. 2, except for UVO 0512-08.

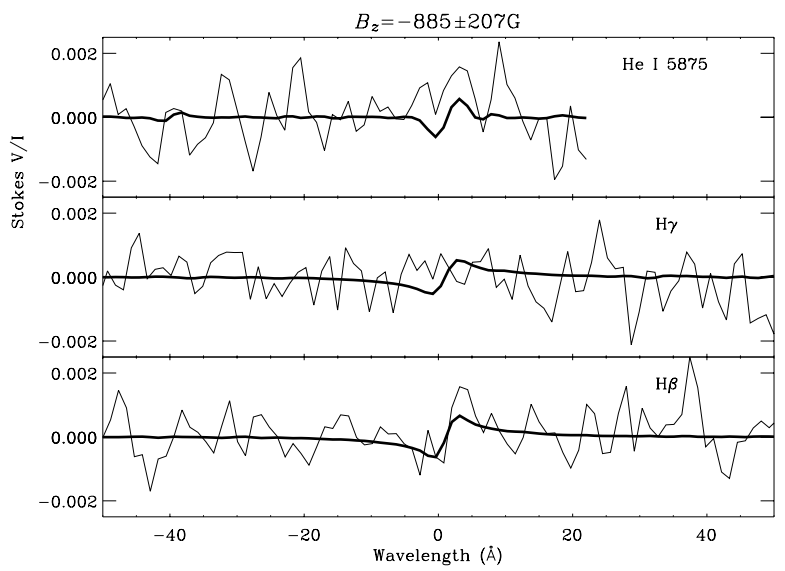

Fig. 4. Similar to Fig. 2, except for $\mathrm{CPD}-64^{\circ} 481$. The top panel shows the He I $5875 \AA$ line at the edge of the spectrum.

$B_{z}=-1448 \pm 222 \mathrm{G}$ for PG $0909+276$. Both of these results are statistically significant (see the previous section), and are also the two highest field strengths we have measured. Whether or not this is physically significant remains to be seen, since the two "normal" sdBs we have observed may have evolved differently (as a binary system), or have evolved well away from the EHB. The super-metal-rich stars are both apparently single, although with its low surface gravity and high temperature, UVO 0512-08 appears to have also evolved away from the EHB. 


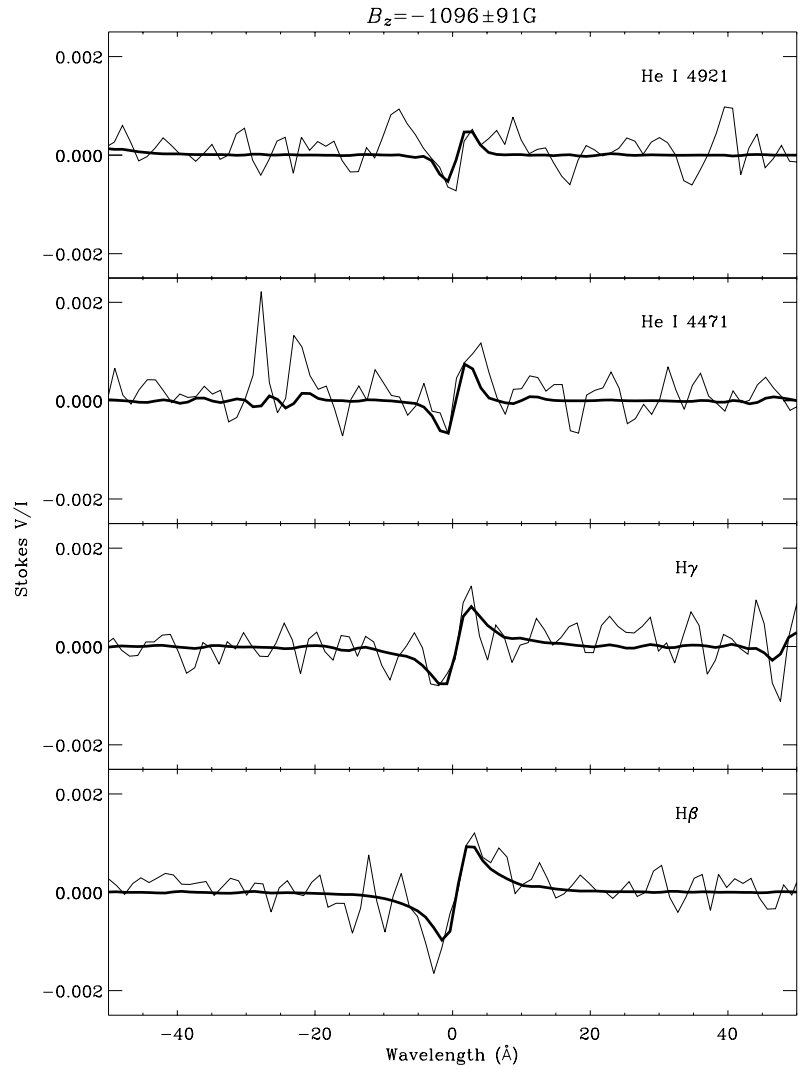

Fig. 5. Similar to Fig. 2, except for HD 76431. In this star the polarisation signature is evident in every line.

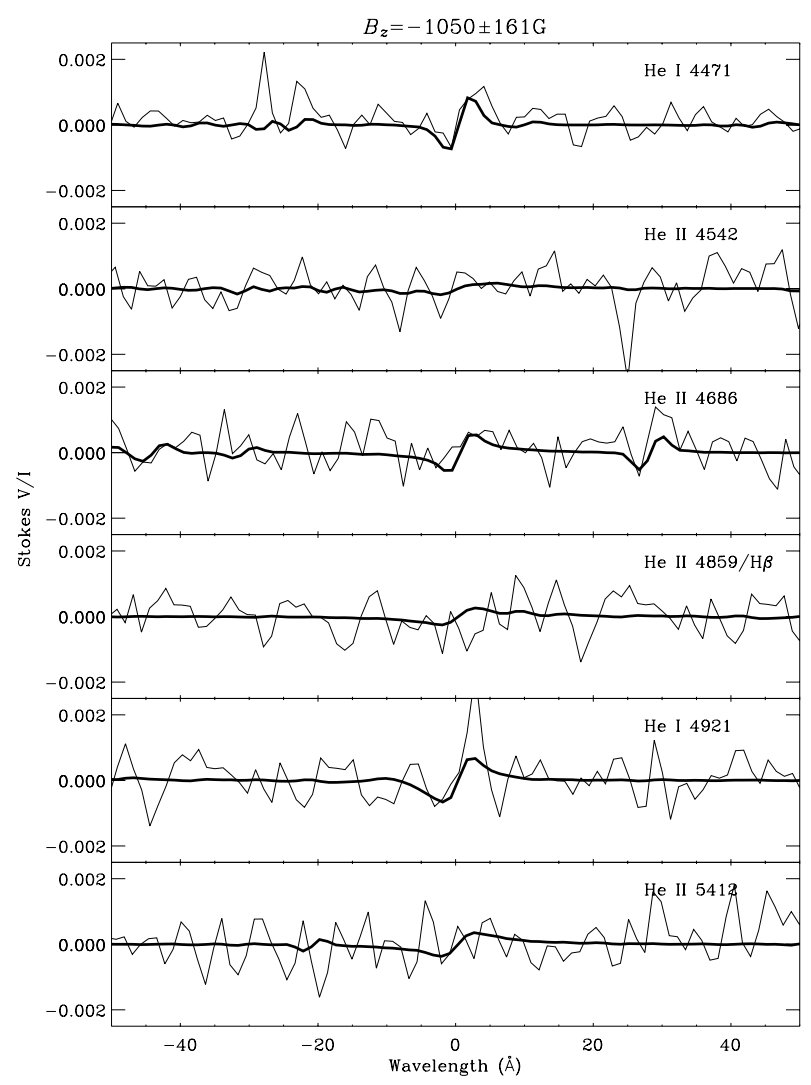

Fig. 6. Similar to Fig. 2, except for $C D-31^{\circ} 4800$.

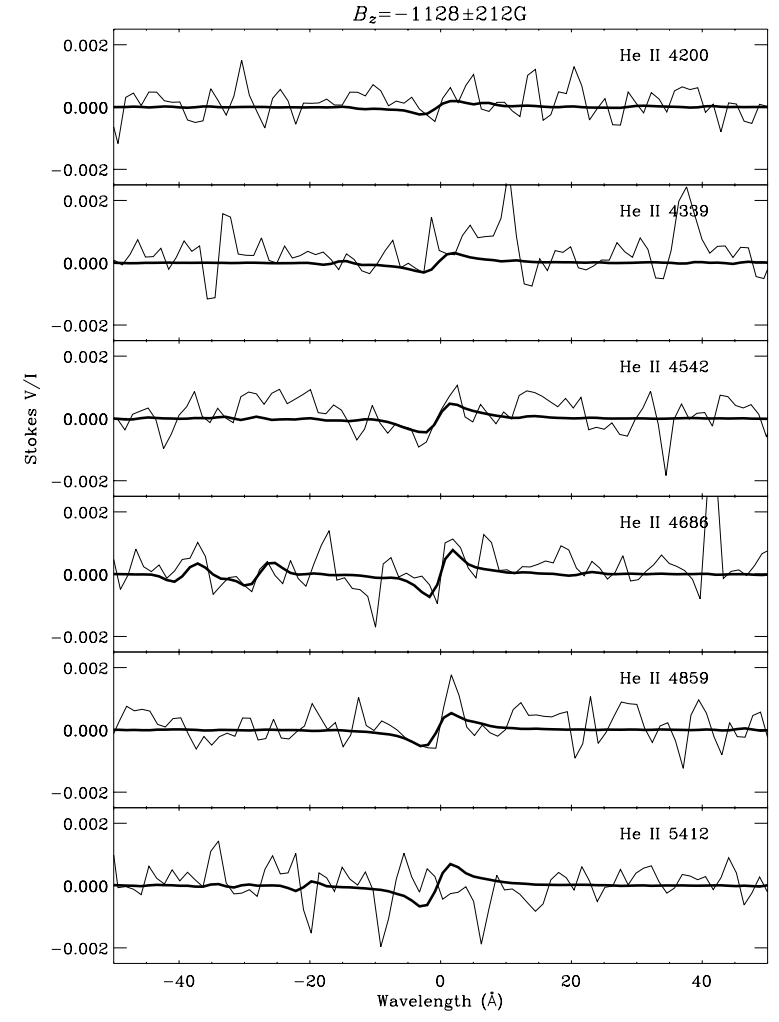

Fig. 7. Similar to Fig. 2, except for LSE 153.

\section{3. $C P D-64^{\circ} 481$ - a typical $s d B$ star}

$\mathrm{CPD}-64^{\circ} 481$ is an $\mathrm{sdB}$ in a short-period $(0.27 \mathrm{~d})$ binary with an unseen companion of low mass, possibly a brown dwarf (Edelmann et al., in preparation). This star has the lowest measured field and the measurement with the lowest significance, with $B_{z}=-885 \pm 207 \mathrm{G}$, however, the probability for an almost kilogauss field is still very high. CPD $-64^{\circ} 481$ had the smallest number of spectral lines available to measure polarisation. We are uncertain how binary evolution - most likely the system has passed through a common envelope phase - might affect the magnetic field of the star's progenitor.

\subsection{HD 76431 - an evolved sdB star}

HD 76431 was originally classified as a main sequence star at high galactic latitude. Its subluminous nature was discovered by a detailed quantitative spectral analysis by Ramspeck et al. (2001). The helium deficiency and a peculiar metal abundance is similar to that of the $\mathrm{sdB}$ stars, although the deviation from solar values are smaller than typical for these stars. The gravity of HD 76431 is slightly higher than that of a main sequence star, but considerably lower than for EHB stars. Comparison to EHB evolutionary tracks suggests that it has already left the EHB and is evolving towards the white dwarf cooling sequence as has been suggested for some sdO stars. Therefore HD 76431 is not a typical sdB, but might be considered as a link between the sdB and sdO stars. The magnetic field measured $\left(B_{Z}=-1096 \pm 91 \mathrm{G}\right)$ has very high significance as found in Sect. 4.1. This star has the clearest "by eye" polarisation signature. 


\subsection{Helium-rich sdOs}

Both of the sdO stars we observed are helium-rich. For CD $-31^{\circ} 4800$ Bauer \& Husfeld (1995) found $T_{\text {eff }}=44000 \mathrm{~K}$ and $\log g=5.9$ and measured abundances; the star contains $\sim 30$ time more helium then hydrogen, and appears to be a "garden variety" sdO (see, e.g. Ströer et al. 2005). We measure $B_{z}=-1050 \pm 161 \mathrm{G}$ based on the 11 lines shown in Table 3, and find from our simulations that this value is statistically significant.

LSE 153 is an extremely hydrogen-deficient sdO that has been suggested as a possible successor of a $\mathrm{R} \mathrm{CrB} \mathrm{star,} \mathrm{with} \mathrm{a}$ mass of $\sim 0.7 M_{\odot}$ (Husfeld et al. 1989). The star's very low hydrogen abundance is accompanied by both nitrogen and carbon enhancements. The field strength we measure $\left(B_{z}=-1128 \pm\right.$ $212 \mathrm{G}$ ) was also found to be statistically significant. This star is at a similar evolutionary stage as the central stars of planetary nebulae studied by Jordan et al. (2005), and has a field strength of approximately the same order of magnitude.

In both of the helium-rich sdOs we have observed the magnetic field determined using the He II 5412 line does not match the other He II lines. We cannot see any obvious reason for this strange behaviour.

\section{Discussion}

The goal of our project was to determine whether magnetic field strength is directly connected to abundance anomalies in these stars. The magnetic field could have an influence on the chemical composition by suppressing convection or by influencing mass-loss or diffusion. While convection is not expected to be important in helium-poor sdB stars, He II ionisation can initiate convection in helium-rich sdO stars at $T_{\text {eff }}$ between 40000 and $50000 \mathrm{~K}$ (Groth et al. 1985). This may be relevant for $\mathrm{CD}-31^{\circ} 4800$, but not for the other programme stars. However, no clear differences exist between the field strengths of the "normal" and super metal-rich stars in our sample.

The origin of the fields detected here are unknown, and are found in objects from various evolutionary channels. It is possible that they are dynamo induced or a remnant from their progenitor. We consider a dynamo-induced magnetic field may be possible if rapid internal rotation occurs as suggested recently by Kawaler \& Hostler (2005), who found evidence that several of the $\mathrm{sdB}$ pulsators may have rapidly rotating cores. This is contrasted with the stars' slow surface rotation (typically $v \sin i \leq 5 \mathrm{~km} \mathrm{~s}^{-1}$, e.g. Heber et al. 2000). While the sdBs have probably followed a similar evolutionary path, the same cannot be said for at least one of the sdO stars. LSE 153 can be associated with the "born-again" post-AGB scenario (Husfeld et al. 1989), while the helium-rich $\mathrm{sdO}, \mathrm{CD}-31^{\circ} 4800$, is similar to most other objects of its class.

It is interesting to estimate the strength of the magnetic fields when these stars have evolved to white dwarfs. If we assume complete conservation of magnetic flux the magnetic fields are amplified inversely proportional to the square of the stellar radius. The radii of the sdB stars are $0.15-0.25 R_{\odot}$ (taking the canonical mass of an EHB star to be $0.48 M_{\odot}$ ). Since they will evolve directly into white dwarfs, their radii will shrink by a factor of $\sim 20$, leading to field strengths of up to $500 \mathrm{kG}$, values which are apparently rarely seen. The search for rotation in white dwarfs (Heber et al. 1997; Koester et al. 1998; Karl et al. 2005) from high resolution $\mathrm{H} \alpha$ spectroscopy also resulted in constraints on field strengths. Amongst the 50 DA white dwarfs studied, only four turned out to be magnetic with $B$ up to $180 \mathrm{kG}$, while upper limits of $10-20 \mathrm{kG}$ could be derived for the rest. Aznar Cuadrado et al. (2004) have detected magnetic fields between 2000 and $4000 \mathrm{G}$ in four white dwarfs, while no magnetic fields on this level of accuracy were found in the other eight programme stars. However, since subdwarfs contribute to only a small percentage $(1-2 \%)$ of white dwarfs progenitors, and the current sample of sub-MG white dwarfs is still small, we cannot completely rule out the existence of low-mass $\left(\sim 0.5 M_{\odot}\right)$ magnetic white dwarfs, although the population must not be large. It is much more difficult to work backwards to values one might expect for the main sequence progenitors of our targets, since sdB formation is not well defined. However, if we take a star with the same parameters as the Sun as an example, we find field strengths in the $20-40 \mathrm{G}$ range, which seem reasonable (although currently undetectable). This also appears to rule out any evolutionary connection with the Ap stars.

Jordan et al. (2005) have found kilogauss magnetic fields in all of the central stars of planetary nebula that they have observed by means of spectro-polarimetry. By magnetic field amplification these stars will have magnetic fields strengths between 0.35 and $2 \mathrm{MG}$ if the assumption of complete conservation of magnetic flux is true. The same holds for the helium-rich sdO star LSE 153 analysed in this paper. Since about two thirds of the white dwarfs seem to have magnetic field even lower than $1 \mathrm{kG}$ (Aznar Cuadrado et al. 2004) one must conclude that magnetic fields can be destroyed during the final stages of stellar evolution, although the detailed mechanisms are unknown.

There are several questions that have arisen from our detection of magnetic fields in hot subdwarfs. Firstly, are the field strengths dependent on the binary status of the star? The one star we have observed that is known to be in a binary system has the lowest and a somewhat less statistically significant measurement. Since it is only one object we cannot make any conclusions at this stage, but this may warrant further investigation. Secondly, what effect, if any, might kilogauss magnetic fields have on pulsations in sdB stars? Rotation is often considered in connection to $\mathrm{sdB}$ pulsators, however magnetic fields have not as yet. Asteroseismology has been used to infer a magnetic field in at least one white dwarf (the pulsating DB GD 358, see Winget et al. 1994). This field may be dynamo induced (Markiel et al. 1994). Spectropolarimetric observations of sdB pulsators, along with a theoretical investigation, would help to clarify this situation.

\section{Conclusions}

In this study, we have used polarisation measurements of $\mathrm{sdB}$ and sdO stars to try to determine whether magnetic fields in two super-metal-rich stars can explain their extreme abundance peculiarities. Field strengths of up to $\sim 1.5 \mathrm{kG}$ range have been 
measured at varying levels of significance in each of our six targets, however no clear difference was found between apparently normal subdwarfs and the metal-rich objects. The origin of the magnetic fields is unknown. We also considered the implications of our measurements for magnetic flux conservation in late stages of evolution. Unless there is a population of lowmass white dwarfs with field strengths up to $\sim 500 \mathrm{kG}$, it is unlikely that magnetic flux is conserved in late stages of evolution. This idea is consistent with findings for central stars of planetary nebulae.

Acknowledgements. We would like to thank E. degl'Innocenti for his advice on Landé factors, and Svetlana Hubrig for help with the observational setup. S.J.O.T. is supported by the Deutsches Zentrum für Luft- und Raumfahrt (DLR) through grant No. 50 OR 0202.

\section{References}

Aznar Cuadrado, R., Jordan, S., Napiwotzki, R., et al. 2004, A\&A, 423, 1081

Bagnulo, S., Szeifert, T., Wade, G. A., Landstreet, J. D., \& Mathys, G. 2002, A\&A, 389, 191

Bauer, F. \& Husfeld, D. 1995, A\&A, 300, 481
Casini, R. \& Landi degl'Innocenti, E. 1994, A\&A, 291, 668

Edelmann, H., Heber, U., \& Napiwotzki, R. 2001, Astron. Nachn., 322,401

Elkin, V. G. 1996, A\&A, 312, L5

Groth, H. G., Kudritzki, R. P., \& Heber, U. 1985, A\&A, 152, 107

Heber, U. 1986, A\&A, 155, 33

Heber, U., Napiwotzki, R., \& Reid, I. N. 1997, A\&A, 323, 819

Heber, U., Reid, I. N., \& Werner, K. 2000, A\&A, 363, 198

Husfeld, D., Butler, K., Heber, U., \& Drilling, J. S. 1989, A\&A, 222, 150

Jordan, S., Werner, K., \& O’Toole, S. J. 2005, A\&A, 432, 273

Karl, C. A., Napiwotzki, R., Heber, U., et al. 2005, A\&A, 434, 637

Kawaler, S. D. \& Hostler, S. R. 2005, ApJ, 621, 432

Koester, D., Dreizler, S., Weidemann, V., \& Allard, N. F. 1998, A\&A, 338,612

Landstreet, J. D. 1982, ApJ, 258, 639

Leone, F., Catanzaro, G., \& Catalano, S. 2000, A\&A, 355, 315

Markiel, J. A., Thomas, J. H., \& van Horn, H. M. 1994, ApJ, 430, 834

Ramspeck, M., Heber, U., \& Edelmann, H. 2001, A\&A, 379, 235

Ströer, A., Heber, U., Lisker, T., Napiwotzki, R., \& Dreizler, S. 2005, in White Dwarfs, ed. D. Koester \& S. Moehler, ASP Conf. Ser., in press [arXiv: astro-ph/0410433]

Winget, D. E., Nather, R. E., Clemens, J. C., et al. 1994, ApJ, 430, 839 